TECHNICAL WORKING PAPER SERIES

\title{
ENVIRONMENTAL POLICY AND FIRM BEHAVIOR: ABATEMENT INVESTMENT AND LOCATION DECISIONS UNDER UNCERTAINTY AND IRREVERSIBILITY
}

Anastasios Xepapadeas

Technical Working Paper 243

http://www.nber.org/papers/T0243

\section{NATIONAL BUREAU OF ECONOMIC RESEARCH \\ 1050 Massachusetts Avenue \\ Cambridge, MA 02138 \\ August 1999}

I would like to thank Carlo Carraro, Charles Kolstad, Gilbert Metcalf, Emanuel Petrakis, Jerome Rothenberg, and the rest of the participants in the NBER/FEEM conference on the "Distributional and Behavioral Effects of Environmental Policy" for helpful comments on earlier drafts of this paper. Any opinions expressed are those of the author and not those of the National Bureau of Economic Research.

(C) 1999 by Anastasios Xepapadeas. All rights reserved. Short sections of text, not to exceed two paragraphs, may be quoted without explicit permission provided that full credit, including $\odot$ notice, is given to the source. Environmental Policy and Firm Behavior: Abatement 
Investment and Location Decisions under Uncertainty

and Irreversibility

Anastasios Xepapadeas

NBER Technical Working Paper No. 243

August 1999

\begin{abstract}
This paper explores abatement investment and location responses to environmental policy, which takes the form of emission taxes or tradeable emission permits and subsidies against the costs of abatement investment, under uncertainty and irreversibility. Uncertainty is associated with output price, environmental policy parameters, or technological parameters. Irreversibility is related to abatement expenses and movements to a new location. Uncertainty is modeled by Itô stochastic differential equations, and the problem is analyzed by using optimal stopping methodologies. Continuation intervals during which firms do not engage in abatement investment or relocate and intervals during which firms take the irreversible decision of undertaking abatement expenses or relocating are defined. Free boundaries are characterized for a variety of cases that include output price uncertainty, policy uncertainty expressed both in terms of continuous fluctuations of permit prices and unpredictable policy changes, and combined policy and technological uncertainty. An optimal environmental policy is defined as the combination of policy parameters that makes the free boundary corresponding to the profit maximization problem coincide with the free boundary corresponding to a social optimization problem.
\end{abstract}

Anastasios Xepapadeas

University of Crete

Department of Economics

University Campus

74100 Rethymno

Greece

xepapad@econ.soc.uoc.gr 


\section{Introduction}

A firm's response to changes in environmental policy is an issue that has drawn considerable attention in the environmental economics literature (e.g. [17], [18], [11], [9]). Questions that usually arise when environmental policy is introduced or changed have been associated with how firms react to a change in environmental policy, primarily with regard to their choices of investment in productive or abatement capital, the mix of relatively more or less polluting inputs, the choice of labor input or the decisions about $\mathrm{R} \& \mathrm{D}$ expenses (process R\&D or environmental R\&D)(e.g. [17], [18], [11], [9], [2], [3]), or what kind of decisions firms make regarding the location choices in response to changes in environmental policy (e.g., [13], [14], [15], [10], [16], [4]).

This paper analyses the behavior of polluting firms regarding expansion of abatement capital and location decisions, in the presence of environmental policy. Environmental policy takes the form of emission taxes or tradeable emission permits, and subsidies against the costs of expanding abatement capital. In this model accumulated abatement capital can be interpreted as the stock of knowledge in pollution and abatement processes. This knowledge is useful in designing new 'cleaner' products or better abatement processes.

The problem is analyzed in a dynamic framework and takes into account two basic features of the problem. The first is uncertainty associated with output price movements, environmental policy parameters such as the market price of tradable emission permits, or technological parameters such as the efficiency of the abatement processes. The second is irreversibility related to the fact that abatement investment expenses are indeed irreversible once they are incurred by the firm, and that movement to a new location when the costs of returning to the old location are sufficiently high is also an irreversible decision. In a dynamic set-up the interaction of uncertainty with the irreversibility characteristics of investment decisions or relocation decisions generates well-known option value issues (see [1], [6], [7], [20] for related environmental issues or [5] for a more general treatment).

The purpose of the present paper is to explore abatement investment and location responses to environmental policy under uncertainty and irreversibility. The problem is analyzed in a dynamic set-up, where uncertainty is modeled by Itô stochastic differential equations, by using optimal stopping methodologies. The idea is to define continuation intervals during which firms do not engage in expanding abatement capital or relocate and intervals during which firms take the irreversible decision of undertaking abatement investment expenses or relocating. The optimal stopping methodology will define a free boundary. When a state variable crosses the boundary, the 
irreversible decision, increasing abatement capital or relocating, is taken.

Using this methodological approach, free boundaries are determined or characterized for a variety of cases that include output price uncertainty, policy uncertainty expressed both in terms of continuous fluctuations of permit prices and unpredictable policy changes, and combined policy and téchnological uncertainty. The effects from exogenous parameters changes (potentially policy parameters) on the position of the boundary, which of course implies changes in the firm's reactions, are analyzed. The issue of the design of optimal environmental policy in this framework is also examined. Optimal policy is defined as the combination of policy parameters that makes the free boundary corresponding to the profit maximization problem coincide with the free boundary corresponding to a social optimization problem.

\section{Abatement Investment Decisions under Uncertaintyl}

We assume an industry consisting of $n$ identical firms producing in a small open economy. The firms behave compettively and sell their product in the world market where international competition prevails. We consider the representative firm producing at each instant of time output $q(t)$ at a cost determined by a cost function $c(q(t))$, with $c^{\prime}(q)>0, c^{\prime \prime}(q)>0$. Output is sold in the world market at an exogenous world price $p(t)$.

The production of output generates emissions. Emissions per unit of output are determined by the function $E(t)=v(t) e(R(t))$, where $v(t)>0$ is an efficiency parameter associated with the abatement process and $e(R(t))$ is a function of the accumulated abatement capital, up to time $t .{ }^{1}$ A reduction in $v$ indicates an improvement in the efficiency of the abatement process, while abatement capital, denoted by $R(t)$, is defined as

$$
R(t)=\int_{0}^{t} r(s) d s
$$

where $r(s)$ is the abatement investment flow undertaken at instant of time $s$. This flow can, for example, represent resources devoted to the firm's lab in order to design cleaner processes at time $s$. It is assumed that $r(s) \geq 0$, thus the abatement capital accumulation process is irreversible. ${ }^{2}$ For the abatement process we assume that:

$$
\begin{aligned}
e(0) & =0, e^{\prime}(R)<0, e^{\prime \prime}(R)>0 \\
\lim _{R \rightarrow 0} e^{\prime}(R) & <-\infty, \lim _{R \rightarrow \infty} e^{\prime}(R)=0
\end{aligned}
$$

Therefore an increase in abatement capital reduces unit emissions at a decreasing rate, which means that diminishing returns in abatement capital 
are assumed. Thus, when the firm produces output $q(t)$, total emissions are defined as $v(t) e(R(t)) q(t)$.

The cost for increasing the stock of accumulated abatement capital by $\Delta R$ is defined as $(1-s) h \Delta R$, where $h$ is the exogenous unit abatement investment cost and $s \in[0,1)$ is a subsidy potentially given by the government to cover some of the expenses for expanding abatement capital. Assume that the firm pays an exogenously determined emission tax $\tau(t)$. Then the tax payments are defined as: $\tau(t)[v(t) e(R(t)) q(t)]$.

Given this set-up the firm has to decide about output production and abatement investment. At each instant of time the firm decides about the optimal output level given the stock of abatement capital. Thus output is regarded as an operating variable and output decisions can be regarded as "short-run" decisions while abatement investment decisions are "long-run" decisions. The optimal choice of output for any given level of abatement capital determines a reduced form instantaneous profit function which can be defined as:

$$
\pi(p, v, \tau, R)=\max _{q}[p(t) q(t)-c(q(t))-\tau(t)[v(t) e(R(t)) q(t)]]
$$

The first-order conditions for the optimal output choice, assuming interior solutions and dropping $t$ to simplify notation, are given as:

$$
p-c^{\prime}(q)-\tau v e(R)=0
$$

with optimal output determined as:

$$
q^{*}=q^{*}(p, \tau, v, R)
$$

Using the first-order conditions for the optimal output choice we obtain the following short-run comparative static results:

$$
\frac{\partial q^{*}}{\partial p}>0, \frac{\partial q^{*}}{\partial \tau}<0, \frac{\partial q^{*}}{\partial v}<0, \frac{\partial q^{*}}{\partial R}>0
$$

Thus an increase in the tax rate or a reduction in the abatement efficiency (increase in $v$ ) reduces optimal output, while an increase in the stock of abatement capital increases optimal output. From the short-run comparative statics and the envelope theorem we obtain the derivatives of the profit function as: ${ }^{3}$ 


$$
\begin{aligned}
& \frac{\partial \pi}{\partial p}=q^{*}(p, v, \tau, R), \frac{\partial^{2} \pi}{\partial p^{2}}=\frac{\partial q^{*}}{\partial p}>0 \\
& \frac{\partial \pi}{\partial v}=-\left(\tau e(R) q^{*}\right)<0, \frac{\partial^{2} \pi}{\partial v^{2}}=-\tau e(R) \frac{\partial q^{*}}{\partial v}>0 \\
& \frac{\partial \pi}{\partial \tau}=-\left(v e(R) q^{*}\right)<0, \frac{\partial^{2} \pi}{\partial \tau^{2}}=-v e(R) \frac{\partial q^{*}}{\partial \tau}>0 \\
& \frac{\partial \pi}{\partial R}=-\tau v e^{\prime}(R) q^{*}>0, \frac{\partial^{2} \pi}{\partial R^{2}}=-\tau v\left(e^{\prime \prime}(R) q^{*}+e^{\prime}(R) \frac{\partial q^{*}}{\partial R}\right)
\end{aligned}
$$
Thus the profit function is convex in prices for fixed $(\tau, v, R)$, decreasing in
$(\tau, v)$ and increasing in $R$.

Uncertainty can be introduced into this model in a number of ways:

- It can be assumed that the world demand is affected by stochastic shocks giving rise to a geometric Brownian motion price processes. In this case output price is the exogenous state variable:

$$
d p(t)=a p(t) d t+\sigma p(t) d z_{p}(t)
$$

where $\left\{z_{p}(t)\right\}$ is a Wiener process, ${ }^{4}$ and $a$ and $\sigma$ are constants. If the current price is a given constant $p(0)=p_{o}$, then the expected value of $p(t)$ is $E[p(t)]=p_{o} e^{a t}$ and the variance of $p(t)$ is: ${ }^{5}$

$$
V[p(t)]=p_{o}^{2} e^{2 a t}\left(e^{\sigma^{2} t}-1\right)
$$

It should be noted that the Brownian motion assumption causes price to move away from its starting point. If however price is related to long-run marginal costs then a better assumption about price movements could be a mean-reverting process. Under this assumption price tends towards marginal costs in the long run and price movements can be modeled as

$$
d p(t)=a(\tilde{p}(t)-p(t)) p(t) d t+\sigma p(t) d z_{p}(t)
$$

where $\widetilde{p}(t)$ can be interpreted as long-run marginal costs. ${ }^{6}$

- It can be assumed that environmental efficiency evolves stochastically according to the geometric Brownian motion

$$
d v(t)=\gamma \tau(t) d t+\delta v(t) d z_{v}(t)
$$


The interpretation of this type of uncertainty can be associated with , the stochastic operating conditions of abatement equipment. It can also be associated with stochastic effects of the general level of abatement knowledge in the economy which is external to the firm, but can affect the firm's abatement efficiency though spillover effects. ${ }^{7}$

- It can be assumed that environmental regulation takes place through a system of tradeable permits, in which case $\tau(t)$ can be interpreted as the competitive market price for permits which can evolve stochastically according to the geometric Brownian motion: 8,9

$$
d \tau(t)=\eta \tau(t) d t+\omega \tau(t) d z_{\tau}(t)
$$

Given the firm's instantaneous profit function (1), the next stage is to define the optimal abatement investment policy for the types of uncertainty described above.

\subsection{Abatement investment decisions under price un- certainty}

Having optimally chosen the output level, the next step is to analyze the decision to undertake new abatement investment, denoted by $\Delta R$, from the existing abatement capital level of $R_{0}$, under price uncertainty modeled by (2) and assuming that $(\tau, v, s, h)$ are fixed parameters. Consider the firm's decision to undertake new abatement investment by $\Delta R$ from the existing abatement capital level $R_{0}$ then, the new abatement capital level becomes:

$$
R_{0^{+}}=R_{0}+\Delta R
$$

The cost of this change in abatement capital is defined as:

$$
(1-s) h\left(R_{0^{+}}-R_{0}\right)
$$

In the model developed here, the optimal abatement investment strategy takes the form of a free boundary, $p=p(R ; \tau, v, s, h)$, relating price and accumulation of abatement capital. This boundary is parametrically defined for the vector of parameters $(\tau, v, s, h)$. When observed price $p^{\text {ob }}$ is less than $p(R ; \tau, v, s, h)$, no abatement investment is undertaken, while when $p^{\mathrm{ob}}$ is greater than $p(R ; \tau, v, s, h)$ enough abatement investment is undertaken in the current period to restore equality on the boundary. Changes in the parameter vector $(\tau, v, s, h)$ shift the boundary and can accelerate or decelerate abatement investment accumulation for any given price. 
Assume that the initial price is $p_{0}$ and the firm's initial abatement capital stock is $R_{0}$. Given a discount rate $\rho$, the firm seeks the nondecreasing process $R(t)$, which will maximize the present values of profits less the cost of development. The value function associated with this problem can be written as:

$$
V(p, R)=\max _{R} \mathcal{E} \int_{0}^{\infty} e^{-\rho t} \pi(p(t), v(t), \tau(t), R(t)) d t
$$

By the concavity of the profit function in $R$, the value function is also concave in $R$ [5].

At each instant of time the firm has two choices: to undertake the new abatement investment or not. The time interval when no new abatement investment is undertaken and the existing abatement stock is used to determine the unit emission coefficient, can be defined as the continuation interval. A stopping time is defined as a time $\mathcal{T}$ at which new abatement investment is undertaken.

et $R^{*}(\mathcal{T})$ be the optimal development process at time $\mathcal{T}$. If $\mathcal{T}$ is a stopping time, then $[8]$ :

$$
\begin{aligned}
V(p, R ; \tau, v)= & \max _{R} \mathcal{E}\left[\int_{0}^{\mathcal{T}} e^{-\rho u} \pi(p(u), v, \tau, R(u)) d u\right. \\
& \left.+e^{-\rho \mathcal{T}} V\left(R^{*}(\mathcal{T}), p(\mathcal{T})\right)\right]
\end{aligned}
$$

where $R^{*}(\mathcal{T})$ is the optimal process at time $\mathcal{T}$. Assume that in the time interval $[0, \theta]$, the firm undertakes no new abatement investment, but keeps it constant at $R_{0}$. By the principle of dynamic programming, the value function should be no less than the continuation payoff in the interval $[0, \theta]$, plus the expected value after $\theta$, or:

$$
\begin{aligned}
V(p, R ; \tau, v) \geq & \mathcal{E}\left[\int_{0}^{\theta} e^{-\rho u} \pi(p(u), v(u), \tau(u), R(u)) d u\right. \\
& \left.+e^{-\rho \theta} V(R(\theta), p(\theta))\right]
\end{aligned}
$$

with equality if $R_{0}$ is the optimal policy in $[0, \theta]$. Applying Itô's lemma to the value function on the right hand side of (8), dividing by $\theta$ and taking limits as $\theta \rightarrow 0$, we obtain: ${ }^{10}$ 


$$
\rho V \geq \frac{1}{2} \sigma^{2} p^{2} V_{p p}+a p V_{p}+\pi(p, v, \tau, R)
$$

with equality if $R(t)=R_{0}$ in the interval $[0, \theta]$.

Consider now the decision to undertake abatement investment instantaneously by $\Delta R=R_{0^{+}}-R_{0}$. Then from the definition of the optimal stopping time, we have

$$
V(p, R ; \tau, v) \geq \mathcal{E}\left[V\left(R_{0^{+}}, p ; \tau, v\right)-(1-s) h\left(R_{0^{+}}-R_{0}\right)\right]
$$

Since the value function is concave in $R$, the optimal abatement investment flow can be obtained by maximizing the right hand side of (10). The necessary and sufficient conditions for the optimal abatement investment choice is:

$$
\begin{aligned}
& V_{R}(R, p ; \tau, v)-(1-s) h \leq 0, \\
& \text { with equality if } \Delta R>0
\end{aligned}
$$

Thus when no new abatement investment is optimal, (9) is satisfied as equality, while when new abatement investment is optimal, (11) is satisfied as equality. Combining (9) and (11), the Hamilton-Jacobi-Bellman (HJB) equation can be written as:

$$
\min \left\{\left[\rho V-\frac{1}{2} \sigma^{2} p^{2} V_{p p}-a p V_{p}-\pi(p, v, \tau, R)\right],-\left[V_{R}-(1-s) h\right]\right\}=0
$$

The optimal free boundary will divide the $(p, R)$ space into two regions: the "no new abatement investment" region, which we will call Region I, and the "new abatement investment" region, which we will call region II.

In region I the first term of the HJB equation (12) is zero, since $\Delta R=0$, and the second term of the HJB equation is positive by (11), thus

$$
\rho V-\frac{1}{2} \sigma^{2} p^{2} V_{p p}-a p V_{p}-\pi(p, R ; \tau, v)=0
$$

The general solution of this second-order differential equation (13) can be obtained as: ${ }^{11}$ 


$$
V(p, R)=A_{1}(R) p^{\beta_{1}}+A_{2}(R) p^{\beta_{2}}+\Pi(p, R ; \tau, v)
$$

where $\beta_{1}=\frac{1}{2}-\frac{a}{\sigma^{2}}+\sqrt{\left(\frac{a}{\sigma^{2}}-\frac{1}{2}\right)^{2}+\frac{2 \rho}{\sigma^{2}}}>1$ is the positive root, and $\beta_{2}$ is the corresponding negative root of the fundamental quadratic:

$$
Q=\frac{1}{2} \sigma^{2} \beta(\beta-1)+a \beta-\rho=0,
$$

and $\Pi(p, R ; \tau, v)$ is the particular solution. We need to disregard the negative root in order to prevent the value from becoming infinitely large when the price tends to zero, thus we set $A_{2}(R)=0$ (see [5]). So the solution becomes:

$$
V(p, R)=A_{1}(R) p^{\beta_{1}}+\Pi(p, R ; \tau, v)
$$

In order to obtain tractable results we need a better specification of the particular solution. To obtain such a specification, we consider a quadratic cost function $c(q)=\frac{1}{2} c q^{2}$, then the profit function becomes:

$$
\pi(p, \tau, v, R)=\frac{1}{2 c}\left[p^{2}-2 \tau v e(R) p+[\tau v e(R)]^{2}\right]
$$

Using the method of undetermined coefficients we obtain the particular solution as:

$$
\begin{aligned}
& \Pi(p, R ; \tau, v)=\Gamma_{0}+\Gamma_{1} p+\Gamma_{2} p^{2} \\
& \Gamma_{0}=-\frac{[\tau v e(R)]^{2}}{2 c \rho}, \Gamma_{1}=-\frac{\tau v e(R)}{c(a-\rho)}, \Gamma_{2}=\frac{1}{2 c\left(\sigma^{2}+2 a-\rho\right)}
\end{aligned}
$$

In region II the second term of (12) is satisfied as zero and $\Delta R>0$ or,

$$
V_{R}(p, R)-(1-s) h=0
$$

Solving (17) for $p$ in terms of $R$, we can write the yet unspecified boundary equation as: $p=p(R)$. From (14), (15), (16) and (17) we can determine the unknown functions $A_{1}(R)$ and $p=p(R)$ using the 'value matching' and the 'smooth pasting' conditions. ${ }^{12}$

The value matching condition means that on the boundary separating the two regions the two value functions should be equal. Then we have, combining (14) and (17) and substituting for $p$ :

$$
V_{R}(p, R)=A_{1}^{\prime}(R) p^{\beta_{1}}+\Gamma_{0}^{\prime}(R)+\Gamma_{1}^{\prime}(R) p=(1-s) h, p=p(R)
$$


The smooth pasting condition means that the derivatives of the value functions with respect to $p$ on the boundary are equal, or:

$$
V_{R p}(p, R)=\beta_{1} A_{1}^{\prime}(R) p^{\beta_{1}-1}+\Gamma_{1}^{\prime}(R)=0, p=p(R)
$$

Combining (18) and (19) we can solve for the unknown functions $p(R)$ and $A_{1}^{\prime}(R)$ to obtain:

$$
\begin{aligned}
p(R) & =\frac{\beta_{1}}{\beta_{1}-1} \frac{(1-s) h-\Gamma_{0}^{\prime}(R)}{\Gamma_{1}^{\prime}(R)} \\
A_{1}^{\prime}(R) & =-\left(\frac{\Gamma_{1}^{\prime}(R)}{\beta_{1}}\right)[p(R)]^{1-\beta_{1}}
\end{aligned}
$$

Relationship (20) is the equation of the free boundary which can be written, after substituting for $\Gamma_{0}^{\prime}(R)$ and $\Gamma_{1}^{\prime}(R)$, as:

$$
p(R)=\frac{-\beta_{1}}{\beta_{1}-1} \frac{(a-\rho)}{\rho} \frac{\left[c \rho(1-s) h+\tau v e(R) e^{\prime}(R)\right]}{\tau v e^{\prime}(R)}
$$

Since $p(R)>0$ the free boundary is defined for parameter values such that $c \rho(1-s) h>\left|\tau v e(R) e^{\prime}(R)\right|$. In order to describe the free boundary we have, by the assumptions on the unit emission function, $p(0)>0$ and $\lim _{R \rightarrow \infty} p(R)=+\infty$. Furthermore

$$
\frac{\partial p}{\partial R}=\frac{-\beta_{1}}{\beta_{1}-1} \frac{(a-\rho)}{\rho} \frac{\left[(\tau v)\left(e^{\prime}\right)^{3}-c \rho(1-s) h e^{\prime \prime}\right]}{\tau v\left(e^{\prime}\right)^{2}}>0
$$

The free boundary is shown in figure 1. For any given level of abatement capital, random price fluctuations move the point $(R, p)$ vertically up or down. If the point goes above the boundary, then new abatement investment is immediately undertaken so that the point shifts on the boundary. Thus optimal abatement capital accumulation proceeds gradually. In the terminology of Dixit and Pindyck [5], this is a 'barrier control' policy.

\section{[Figure 1]}

By inverting (22) we can obtain the optimal boundary function $R^{*}=$ $p^{-1}(p ; \mathbf{z})$, which determines the optimal abatement investment boundary as a function of the state variable $p$ and the vector $\mathbf{z}$ of the parameters of the problem. For price movements to the right of the boundary, new abatement 
investment is undertaken. If price stays on the left of the boundary, no new , abatement investment is undertaken.

If price follows a mean reverting process then the HJB equation for region I that corresponds to (13) becomes

$$
\rho V-\frac{1}{2} \sigma^{2} p^{2} V_{p p}-a(\widetilde{p}-p) p V_{p}-\pi(p, R ; \tau, v)=0
$$

The steps for solving for the optimal boundary are the same as above, however due to the more complicated structure of $\left(13^{\prime}\right)$ the effects of mean reversion require numerical solutions (see [5]).

\subsection{The impact of changes in policy parameters}

We examine the shifts of the free boundary in response to changes in the tax parameter $\tau$. To examine the effects of a change in the tax policy, we have

$$
\frac{\partial p}{\partial \tau}=\frac{\beta_{1}}{\beta_{1}-1} \frac{(a-\rho)}{\rho} \frac{c \rho(1-s) h}{\tau^{2} v e^{\prime}}<0
$$

Thus an increase in the tax rate shifts the boundary downwards and induces more abatement investment for any given price level, as is also shown in figure 1 . In the same way we obtain:

$$
\frac{\partial p}{\partial v}<0
$$

A reduction in abatement efficiency induces more abatement investment for any given price level.

\subsection{Optimal environmental policy}

In the previous section the tax and the subsidy parameters were treated as fixed. The analysis can, however, be extended to analyze the case of an environmental regulator who can choose optimally the policy parameters. Optimal policy choice is considered in the following way. From the previous analysis it is known that the free boundary that determines the profit maximizing abatement investment in equation (22) depends on the tax and subsidy parameters. Consider the case of an environmental regulator that determines a socially optimal free boundary by explicitly taking into account environmental damages. An optimal environmental policy can then be defined by determining the values of the policy parameters such that the profit 
maximizing free boundary will coincide with the socially optimal free boundary, as determined by the environmental regulator. Define a social profit function by

$$
W(p, v, R)=\max _{q}[p(t) q(t)-c(q(t))-D(v(t) e(R(t)) q(t))]
$$

where $D(v(t) e(R(t)) q(t))$ is a strictly increasing and convex damage function. By following the steps of section 2, a free boundary that determines the socially optimal abatement investment under price uncertainty can be defined. Denote it by $p^{s}(R)$, and consider the free boundary defined in (22) as a function of the policy parameters, or $p(R ; \tau, s)$. An optimal environmental policy can be defined as the pair:

$$
\left(\tau^{*}, s^{*}\right): p\left(R ; \tau^{*}, s^{*}\right)=p^{s}(R)
$$

A solution of the form $\tau^{*}=\zeta\left(s^{*}\right)$ will determine the trade off between emission taxes and abatement investment subsidies in the design of environmental policy. ${ }^{13}$

It is interesting to note that under uncertainty and irreversibility the optimal environmental policy equates the privately optimal and the socially optimal free boundaries and not the privately optimal and the socially optimal levels of the choice variables as in the case of optimal policy design under certainty.

\section{Abatement Investment Decisions under En-I vironmental Policy or Abatement Efficiencyl Uncertainty}

When, under fixed prices, the environmental policy uncertainty is present in the form of stochastic evolution of prices for tradeable emission permits, or abatement efficiency is stochastic, the mathematical treatment is similar, although the sources of uncertainty are different. Policy uncertainty can be regarded as uncertainty outside the firm while abatement uncertainty can be regarded as internal to the firm. So although the mathematical results are the same, their interpretation and their policy implications are different.

In the case of policy uncertainty, the Hamilton-Jacobi-Bellman equation can be written as:

$$
\min \left\{\left[\rho V-\frac{1}{2} \omega^{2} \tau^{2} V_{\tau \tau}-\eta \tau V_{\tau}-\pi(\tau, R ; p, v)\right],-\left[V_{R}-(1-s) h\right]\right\}=0
$$


As before the optimal free boundary will divide the $(\tau, R)$ space into two regions: the "no new abatement investment" region (region I), and the "new abatement investment" region (region II).

In region I the first term of the HJB equation is zero, since $\Delta R=0$, and the second term of the HJB equation is positive by (11), thus

$$
\rho V-\frac{1}{2} \omega^{2} \tau^{2} V_{\tau \tau}-\eta \tau V_{\tau}-\pi(\tau, R ; p, v)=0
$$

The general solution of this second-order differential equation can be obtained as above as:

$$
V(\tau, R)=B_{1}(R) \tau^{\xi_{1}}+B_{2}(R) \tau^{\xi_{2}}+\Phi(p, R ; \tau, v)
$$

where $\xi_{1}=\frac{1}{2}-\frac{\eta}{\omega^{2}}+\sqrt{\left(\frac{\eta}{\omega^{2}}-\frac{1}{2}\right)^{2}+\frac{2 \rho}{\omega^{2}}}>1$ is the positive root and $\xi_{2}$ is the corresponding negative root of the fundamental quadratic

$$
Q=\frac{1}{2} \omega^{2} \xi(\xi-1)+\eta \xi-\rho=0
$$

and $\Phi(\tau, R ; p, v)$ is the particular solution. As before we set $B_{2}(R)=0$, so the solution becomes:

$$
V(\tau, R)=B_{1}(R) \tau^{\xi_{1}}+\Phi(\tau, R ; p, v)
$$

Using again the quadratic cost function specification, $c(q)=\frac{1}{2} c q^{2}$, we obtain the particular solution as:

$$
\begin{aligned}
& \Phi(\tau, R ; p, v)=\Delta_{0}+\Delta_{1} \tau+\Delta_{2} \tau^{2} \\
& \Delta_{0}=-\frac{p^{2}}{2 c \rho}, \Delta_{1}=-\frac{p v e(R)}{c(\eta-\rho)}, \Delta_{2}=\frac{[v e(R)]^{2}}{2 c\left(\omega^{2}+2 \eta-\rho\right)}
\end{aligned}
$$

In region II the second term of the HJB equation is satisfied as zero and $\Delta R>0$, or,

$$
V_{R}(\tau, R)-(1-s) h=0
$$

The value matching and smooth pasting conditions imply that:

$$
V_{R}(\tau, R)=B_{1}^{\prime}(R) \tau^{\xi_{1}}+\Delta_{1}^{\prime}(R) \tau+\Delta_{2}^{\prime}(R) \tau^{2}=(1-s) h, \tau=\tau(R)
$$


and

$$
V_{R \tau}(\tau, R)=\xi_{1} B_{1}^{\prime}(R) \tau^{\xi_{1}-1}+\Delta_{1}^{\prime}(R)+2 \Delta_{2}^{\prime}(R) \tau=0, \tau=\tau(R)
$$

respectively, where

$$
\Delta_{1}^{\prime}(R)=-\frac{\rho v e^{\prime}(R)}{c(\eta-\rho)}>0, \Delta_{2}^{\prime}(R)=\frac{2 v e(R) e^{\prime}(R)}{2 c\left(\omega^{2}+2 \eta-\rho\right)}
$$

Combining (23) and (24) we obtain a quadratic expression which implicitly defines $\tau(R)$ as:

$$
\Delta_{2}^{\prime}(R)\left(\frac{\xi_{1}-2}{\xi_{1}}\right) \tau(R)^{2}+\Delta_{1}^{\prime}(R)\left(\frac{\xi_{1}-1}{\xi_{1}}\right) \tau(R)-(1-s) h=0
$$

By taking the positive root of (25) the free boundary is defined as:

$$
\begin{aligned}
\tau(R) & =\frac{-\xi_{1}}{2\left(\xi_{1}-2\right)} \frac{\Delta_{1}^{\prime}(R)\left(\frac{\xi_{1}-1}{\xi_{1}}\right)+\sqrt{\Delta}}{\Delta_{2}^{\prime}(R)} \\
\Delta & =\left[\Delta_{1}^{\prime}(R)\left(\frac{\xi_{1}-1}{\xi_{1}}\right)\right]^{2}+4 \Delta_{2}^{\prime}(R)\left(\frac{\xi_{1}-2}{\xi_{1}}\right)(1-s) h
\end{aligned}
$$

with $\xi_{1}>2$ for $\Delta>0$.

Using the assumptions about the unit emission function we have

$$
\lim _{R \rightarrow 0} \tau(R)=-\infty, \text { and } \lim _{R \rightarrow \infty} \tau(R)=M>0
$$

If the free boundary is monotonic, a property that can be checked by using specific functions, then its graph is shown in figure 2 .

[Figure 2]

An increase of the policy parameter above the boundary induces more abatement investment. By inverting the $\tau(R)$ function, an optimal boundary function for abatement capital accumulation in terms of the policy parameter $\tau$ is defined as:

$$
R^{*}=\tau^{-1}(\tau, \mathbf{z})
$$

Policy uncertainty and abatement efficiency uncertainty can also be examined together by introducing the variable $z=\tau v$, with $\mathcal{E}\left(d z_{\tau} d z_{v}\right)=\rho_{\tau v} d t$. Using the fact that $\frac{\partial^{2} z}{\partial \tau^{2}}=\frac{\partial^{2} z}{\partial v^{2}}=0$ and $\frac{\partial^{2} z}{\partial \tau \partial v}=1$ we obtain

$$
d z=\left(\gamma+\eta+\rho_{\tau v} \delta \omega\right) z d t+\left(\delta d z_{v}+\omega d z_{\tau}\right) z
$$


Thus changes in $z$ have mean and variance

$$
\begin{aligned}
& k_{1}=\gamma+\eta+\rho_{\tau v} \delta \omega \\
& k_{2}=\delta^{2}+2 \rho_{\tau v} \delta \omega+\omega^{2}
\end{aligned}
$$

respectively. Following the same steps as above the HJB equation is defined as:

$$
\min \left\{\left[\rho V-\frac{1}{2} k_{2}^{2} z^{2} V_{z z}-k_{1} z V_{z}-\pi(z, R ; p)\right],-\left[V_{R}-(1-s) h\right]\right\}=0
$$

Then the free boundary can be defined in the context of correlated policy and technological uncertainty, as in the case of policy uncertainty above.

\subsection{Unpredictable policy changes}

Policy uncertainty as analyzed above is associated with continuous fluctuations of the tradeable emission permit price. It is possible, however, for a sudden change in policy due for example to an unexpected - from the firm's point of view - change in the supply of permits, to cause a discontinuous change in their price. In the context of our model this unpredictable change introduces jump characteristics. Thus, while the usual fluctuations in prices are captured by the geometric Brownian motion, the sudden policy change should be captured by a Poisson process. Therefore the price of permits is modelled by a mixed Brownian motion/jump process, or

$$
d \tau=\eta \tau d t+\omega \tau d z_{\tau}+\tau d q^{P}
$$

where $d q^{P}$ is the increment of a Poisson process with mean arrival time of the change in the supply of permits $\lambda$. We further assume that the change in the supply of permits represents an increase, and that this causes a fixed drop in the price $e^{14}$ by a known percentage $\psi \in[0,1]$ with probability 1 , and that $d z_{\tau}$ and $d q^{P}$ are independent.

To analyze this problem the HJB equation is derived by using Itô's lemma for combined Brownian motion/jump process [5]. Then the HJB equation can be written as:

$$
\min \left\{\begin{array}{c}
{\left[\rho V-\frac{1}{2} \omega^{2} \tau^{2} V_{\tau \tau}-\eta \tau V_{\tau}-\pi(\tau, R ; p, v)\right]+\lambda[V((1-\psi) \tau)-V]} \\
,-\left[V_{R}-(1-s) h\right]
\end{array}\right\}=0
$$

The solution to the value function is

$$
V(\tau, R, \psi)=B_{1}^{\psi}(R) \tau^{\xi_{1}^{\psi}}+\Phi^{\psi}(\tau, R ; p, v, \psi)
$$


where $\xi_{1}^{\psi}$ is the positive solution of the nonlinear equation (see [5]):

$$
\frac{1}{2} \omega^{2} \xi^{\psi}\left(\xi^{\psi}-1\right)+\eta \xi^{\psi}-(\rho+\lambda)+\lambda(1-\psi)^{\xi^{\psi}}=0
$$

Once a solution for $\xi_{1}^{\psi}$ is obtained, then the free boundary can be obtained as before.

It should be noted that special cases of the general mixed Brownian motion/jump process model can be used to analyze specific cases. For example if $\eta=\omega=0$ is set, and $\tau$ is interpreted as an emission tax, then the same model can be used to analyze the implications of unpredictable changes in the emission tax rates.

\section{Location Decisions}

When we examine location decisions, the problem can be defined as an optimal stopping problem. In the waiting or continuation region the firm stays in its present location, pays the emission tax and follows the optimal abatement capital accumulation path, $R^{*}(t)$, given uncertainty described by the evolution of the state variable (price, policy parameter, or technology parameter).

Suppose that the firm examines the possibility of relocation to a new location (country) where there is no environmental policy. Assume that the set-up costs are fixed, $F$, and are incurred once at the time of relocation, that the cost function remains the same and that there are no transportation costs. ${ }^{15}$ Suppose that relocation takes place at time $t_{d}$, then the profit function for the firm that chooses optimally operating output, is defined as:

$$
\left\{\begin{array}{c}
\pi_{d}(p(t))-F, \text { for } t=t_{d} \\
\pi_{d}(p(t)), \text { for } t>t_{d}
\end{array}\right.
$$

where $\pi_{d}(p)=\max _{q}[p q-c(q)]$.

Assuming that price uncertainty exists, then at each period of time the firm faces a binary choice:

1. Relocate and take the termination payoff defined as $\left.W\left(p\left(t_{d}\right), F\right)\right)=$ $\mathcal{E} \int_{t_{d}}^{\infty} e^{-\rho t} \pi_{d}(p(t)) d t-F$.

2. Continue operation at the initial location for one period, choosing output and abatement investment optimally, receive the operating profits and then consider another binary choice in the next period. 
The Bellman equation for this problem can be written as:

$$
V(p)=\max \left\{\pi\left(p, \tau, v, R^{*}\right)+\frac{1}{1+\rho d t} \mathcal{E}[V(p+d p \mid p)], W(p, F)\right\}
$$

In the continuation region, the first term on the right hand side is the largest. Using Itô's lemma on this term we obtain the usual differential equation

$$
\rho V-\frac{1}{2} \sigma^{2} p^{2} V_{p p}-a p V_{p}-\pi\left(p ; R^{*}, \tau, v\right)=0
$$

with solution

$$
V\left(p(t) ; R^{*}, \tau, v\right)=K_{1} p(t)^{\beta_{1}}+\Pi\left(p(t) ; R^{*}, \tau, v\right)
$$

From the Bellman equation we have that at the critical relocation time $t_{d}$ :

$$
V\left(p\left(t_{d}\right) ; R^{*}, \tau, v\right)=W\left(p\left(t_{d}\right), F\right)
$$

This is the value matching condition. The smooth pasting condition requires that:

$$
V_{p}\left(p\left(t_{d}\right) ; R^{*}, \tau, v\right)=W_{p}\left(p\left(t_{d}\right), F\right)
$$

Conditions (29) and (30) can be used to determine the constant $K_{1}$ and the free boundary $p=p^{*}\left(t_{d}\right)$. By inverting the boundary equation we obtain the optimal relocation time boundary function $t_{d}^{*}=p^{*-1}(p)$. This boundary determines the critical relocation time as a function of the observed price for given values of the parameters $\tau, v, s$.

Environmental policy uncertainty or abatement efficiency uncertainty can be treated in the same way. Suppose that policy uncertainty exists in the sense of stochastic permit prices. Then following the same steps as above, the free boundary $\tau=\tau^{*}\left(t_{d}\right)$ is defined by the following conditions, using the quadratic cost function:

$$
\begin{aligned}
V\left(\tau(t) ; R^{*}, p, v\right) & =\Lambda_{1} \tau(t)^{\xi_{1}}+\Phi\left(\tau(t) ; R^{*}, p, v\right) \\
V\left(\tau\left(t_{d}\right) ; R^{*}, p, v\right) & =W(F), \text { value matching } \\
W(p, F) & =\int_{0}^{\infty} e^{-\rho t} \frac{p^{2}}{2 c} d t-F=\frac{p^{2}}{2 c \rho}-F, p \text { fixed } \\
V_{\tau}\left(\tau\left(t_{d}\right) ; R^{*}, p, v\right) & =W_{\tau}(F)=0, \text { smooth pasting }
\end{aligned}
$$

Using the conditions above, the free boundary $\tau^{*}(t)$ is implicitly defined by:

$$
\frac{-\Phi_{\tau}\left(\tau^{*}(t) ; R^{*}, p, v\right)}{\xi_{1}} \tau^{*}(t)+\Phi\left(\tau^{*}(t) ; R^{*}, p, v\right)=\frac{p^{2}}{2 c \rho}-F
$$


By inverting the boundary function $\tau^{*}\left(t_{d}\right)$, we obtain the optimal relocation time boundary function $t_{d}^{*}=\tau^{*-1}(\tau)$ in terms of the environmental policy parameter and the rest of the parameters of the problem. Optimal relocation implies the existence of a threshold policy parameter such that when the actual policy parameter crosses this threshold, then relocation takes place. This result is stated in the following proposition.

Proposition 1 Let $\tau^{\circ}\left(t^{\prime}\right)$ be the observed environmental policy parameter at time $t^{\prime}$. If $\tau^{o}\left(t^{\prime}\right)<\tau^{*}\left(t^{\prime}\right)$, then it is optimal to remain at the initial site. If $\tau^{o}\left(t^{\prime}\right)>\tau^{*}\left(t^{\prime}\right)$ it is optimal to relocate at time $t^{\prime}$.

$P$ roof. e Appendix.

This proposition implies that for any time $t$ a threshold environmental policy parameter exists such that when the policy parameter exceeds the threshold, then the firm moves to the new location. The relation between the threshold policy parameter and the optimal relocation time is shown in figure 3 , where $\bar{\tau}$ indicates the permit price that induces immediate relocation. The lower the permit price, the further away the optimal relocation time is. When the permit price crosses the boundary it is optimal to take the irreversible relocation decision.

[Figure 3]

Similar analysis, although with different interpretation, can be applied to the case where uncertainty relates to abatement efficiency, or to correlated policy and abatement uncertainty.

The above analysis suggests that since firms are identical they will delocate simultaneously when the critical time arrives. If firms are heterogenous regarding characteristics of production cost or abatement technologies, then the optimal delocation time will be different across firms. In this case there will more than one boundary like the one depicted in figure 3 . Suppose that a second boundary, $\tau_{j}^{*}\left(t_{d}\right)$, exists and as shown in figure 3 , the two boundaries do not intersect. Then the same permit price $\tilde{\tau}$ implies different optimal delocation times.

\subsection{Relocation time policy under uncertainty}

The free boundaries and the optimal relocation policy functions derived above can be used to provide useful information regarding the effects of exogenous shocks on the critical relocation time and describe a framework for designing a policy that could affect relocation time. This can be obtained by performing comparative static analysis of the optimal boundary function. 
Assuming again identical firms to simplify things, consider the boundary function $t_{d}^{*}=p^{*-1}(p ; \tau, v, s)$ and take the derivative:

$$
\frac{\partial t_{d}^{*}}{\partial \tau}=\frac{\partial p^{*-1}(p)}{\partial \tau}
$$

This derivative determines the effect on the critical relocation time of an exogenous change in the emission tax for any given price level. In the same way the effects from changes of other parameters of the model on relocation time can also be defined.

Consider now the total differential

$$
d t_{d}^{*}(p)=\frac{\partial t_{d}^{*}}{\partial \tau} d t_{d}+\frac{\partial t_{d}^{*}}{\partial s} d s
$$

This differential can express the rate of substitution between the emission tax rate and the abatement investment subsidy rate in order to produce a given change in the critical relocation time at any given price level. For example by setting $d t_{d}^{*}(p)=0$, the marginal rate

$$
\frac{d s}{d \tau}=-\frac{\frac{\partial t_{d}}{\partial \tau}}{\frac{\partial t_{d}}{\partial s}}
$$

expresses the necessary increase in the abatement investment subsidy in order to keep the critical relocation time constant after an increase in the emission tax for any given price. The changes in the policy parameters shift the free boundary $p=p^{*}\left(t_{d}\right)$ and enlarge or shrink the "stay" or "delocate" regions as shown in figure 4 .

[Figure 4]

The particular forms of the policy functions, the comparative static derivatives, as well as the marginal rates of substitution, can be explored under the quadratic cost function assumption.

\section{Concluding remarks}

The responses of firms to environmental taxation regarding their abatement investment and location decisions were analyzed in an analytical framework characterized by uncertainty and irreversibilities.

The optimal stopping time methodology adopted in this paper makes possible the derivation of optimal policy functions for abatement investment 
and location decisions as functions of prices, environmental policy or abatement efficiency. These policy functions can provide very useful information to a regulator seeking to explore the effects of regulation on firms' decisions. It is also possible to use the optimal stopping time methodology in order to design optimal environmental policy under uncertainty and irreversibility, in the sense of choosing the policy parameters so that the free boundary, or equivalently the optimal policy function under profit maximization, coincides with the socially optimal free boundary (abatement investment policy function).

The implication of this approach regarding policy design under price uncertainty and irreversibility is that a regulator can in principle design a policy scheme consisting of two instruments, an emission tax or a tradable permit system and a subsidy on abatement investment. The policy scheme takes into account uncertainty through its dependence on the parameters on the price process and will induce individual firms to undertake the same output and abatement investment under uncertainty that a regulator would have undertaken. In this sense the policy mix of emission taxes (or emission permits) and abatement investment subsidies will be welfare maximizing. It should be noticed that the function $\tau^{*}=\zeta\left(s^{*}\right)$ determining the optimal trade-off between taxes and subsidies allows the regulator to determine the policy mix in order to obtain an optimal balance between the output contacting pollution control by emission taxes and pollution control throught the subsidization of the accumulation of abatement capital.

A similar mix of emission taxes (or emission permits) and abatement investment subsidies can be used to affect location decisions. By linking location decisions to subsidies in emission-reducing abatement investment, it was possible to derive rules relating to the amount of subsidy required in order not to accelerate relocation after the introduction of a stricter environmental policy. Given the function that determines optimal relocation time as a function of the observed price an increase in emission taxes may induce delocation of all or a subset of firms, depending on the heterogeneity assumptions, by bringing delocation time forward at the same price level. If delocation is not desirable it may be prevented by an appropriate increase in the abatement subsidy. On the other hand if price movements in the world market induce delocation, our results indicate that it could be prevented by an appropriate change of the policy mix, that is changes in emission taxes and/or abatement subsidies.

Further research could be directed towards the study of the relocation time when the country abroad follows a different environmental policy, or when the firms in the home country are heterogenous. Further research could also be directed towards the study of the socially optimal relocation 
time. The optimal stopping time methodology could indicate the time that is socially desirable for a firm to relocate, then help to design a policy scheme to prevent suboptimal relocation decisions. 


\section{Appendix}

\section{$P$ roof. oposition 1}

The Bellman equation is:

$$
V(\tau)=\max \left\{\pi\left(\tau ; p, v, R^{*}\right)+\frac{1}{1+\rho d t} \mathcal{E}[V(\tau+d \tau \mid \tau)], W(p, F)\right\}
$$

Following [5], define $G(\tau)=V(\tau)-W, \tau+d \tau=\tau^{\prime}$ and subtract $W$ from both sides of the Bellman equation above to obtain

$$
\begin{aligned}
G(\tau) & =\max \left\{0, \pi(\tau)-W+\frac{1}{1+\rho d t} \int V\left(\tau^{\prime}\right) d \Phi\left(\tau^{\prime} \mid \tau\right)\right\} \\
& =\max \left\{\begin{array}{c}
0, \pi(\tau)-W+\frac{1}{1+\rho d t} W+ \\
\frac{1}{1+\rho d t} \int G\left(\tau^{\prime}\right) d \Phi\left(\tau^{\prime} \mid \tau\right)
\end{array}\right\}
\end{aligned}
$$

Since $\mathrm{W}$ does not depend on $\tau$, we have that the expression

$$
m(\tau)=\pi(\tau)-W+\frac{1}{1+\rho d t} W
$$

is decreasing in $\tau$, since $\frac{d m}{d \tau}=\frac{d \pi}{d \tau}<0$. The function $m(\tau)$ reflects the difference between waiting for one period before relocating and relocating right away. Since $m(\tau)$ is decreasing in $\tau$, continuation - that is no relocation should be optimal when $\tau$ is low.

Assume that the cumulative distribution $\Phi\left(\tau^{\prime} \mid \tau\right)$ of the future values of the policy parameter shifts uniformly to the left as $\tau$ increases, so that the disadvantages of an increase in the current value of the environmental policy parameter in the original location are unlikely to be reversed in the future. This assumption, along with the decreasing $m(\tau)$ function, implies that $G^{\prime}(\tau)<0$ (see [5] Appendix B).

Therefore the second argument of (32) is decreasing in $\tau$. Thus a unique critical time $\tau^{*}\left(t^{\prime}\right)$ exists such that the second argument of (32) is negative if and only if $\tau^{o}\left(t^{\prime}\right)>\tau^{*}\left(t^{\prime}\right)$. Then it is optimal to relocate (optimal to stop) at time $t^{\prime}$. If $\tau^{o}\left(t^{\prime}\right)<\tau^{*}\left(t^{\prime}\right)$, then it is optimal to remain at the initial site (continue). 


\section{Endnotes}

1 A more general formulation would be to define the $e(\cdot)$ function as $e\left(R, R^{A G}\right)$, where $R^{A G}=n R$, is aggregate abatement knowledge. In this case there could be positive spillovers from aggregate abatement capital to the individual abatement function. When firms consider aggregate knowledge as fixed there is a divergence between the private return of abatement capital and the social return of abatement capital [19].

${ }^{2}$ To simplify things we ignore depreciation issues.

${ }^{3}$ It is assumed that: $\left(e^{\prime \prime}(R) q^{*}+e^{\prime}(R) \frac{\partial q^{*}}{\partial R}\right)>0$, so that the profit function is concave in $R$ for fixed $(p, \tau, v)$. The concavity assumption requires sufficient curvature of the unit emission function $e(R)$.

${ }^{4}$ For definitions see [12].

${ }^{5}$ See [5].

${ }^{6}$ If we consider entry and exit decisions in the world market then an upper reflecting barrier $\bar{p}$ to the price movement can be considered. When price moves to the reflecting barrier, new entry is triggered, quantity increases and price decreases.

${ }^{7}$ Stochastic delays in the R\&D processes can be modelled by assuming that $v$ follows a Poisson process.

${ }^{8}$ Then $v(t) e(R(t)) q(t)$ can be interpreted as the excess demand for permits.

${ }^{9}$ The expected values and the variances for $v(t)$ and of $\tau(t)$ are defined in a similar way as for $p(t)$ above.

${ }^{10}$ Subscripts associated with the value function denote partial derivatives.

${ }^{11}$ The homogeneous part of this differential equation is an Euler equation.

${ }^{12}$ For a presentation of these conditions see [5].

${ }^{13}$ The relationship between the two policy instruments can be further elaborated to include balance-budgeting schemes, where total tax revenues equal total subsidy expenses.

${ }^{14}$ A fixed increase in the price can be treated symmetrically.

${ }^{15}$ Alternative assumptions could include the existence of a different environmental policy abroad, for example command and control regulation, or differences in the political systems that affect the stringency of environmental policy. 


\section{References}

[1] Arrow, K.J and A. Fisher (1974), Environmental Preservation, Uncertainty and Irreversibility, Quarterly Journal of Economics, 88, 312-319.

[2] Carraro, C. and D. Soubeyran (1996a), Environmental Feedbacks and Optimal Taxation in Oligopoly, in A. Xepapadeas (ed.), Economic Policy for the Environment and Natural Resources, Cheltenham, UK: Edward Elgar Publishing, 30-58.

[3] Carraro, C. and A. Soubeyran (1996b), Environmental Policy and the Choice of Production Technology, in C. Carraro, Y. Katsoulacos and A. Xepapadeas (eds), Environmental Policy and Market Structure, Dordrecht: Kluwer Academic Publishers, 151-80.

[4] Carraro, C. and A. Soubeyran, (1998), R\&D Cooperation, Innovation Spillovers and Firm ocation in a Model of Environmental Policy, forthcoming in E. Petrakis, E. Sartzetakis and A. Xepapadeas (Eds.), Environmental Regulation and Market Structure, Edward Elgar, Cheltenham.

[5] Dixit, A.K. and R.S. Pindyck (1994), Investment under Uncertainty, Princeton, New Jersey, Princeton University Press.

[6] Fisher, A.C. and M. Hanemann (1986), Environmental Damages and Option Values, Natural Resource Modelling, 1, 111-124.

[7] Fisher, A.C. and M. Hanemann (1987), Quasi-Option Value: Some Misconceptions Dispelled, Journal of Environmental Economics and Management, 14, 183-190.

[8] Fleming, W. and H. M. Soner (1993), Controlled Markov Process and Viscosity Solutions, Springer-Verlag.

[9] Hartl, R.F. and P.M. Kort (1996), Marketable Permits in a Stochastic Dynamic Model of the Firm, Journal of Optimization Theory and Applications, 89 (1), 129-55.

[10] Hoel, M. (1994), Environmental Policy as a Game between Governments when Plant ocations are Endogenous, 21st EARIE Conference, Crete.

[11] Kort, P.M. (1995), The Effects of Marketable Pollution Permits on the Firm's Optimal Investment Policies, Central European Journal for Operations Research and Economics, 3, 139-55. 
[12] Malliaris, A.G. and W.A. Brock (1982), Stochastic Methods in Economics and Finance, Amsterdam: North-Holland.

[13] Markusen, J.R., E.R. Morey and N. Olewiler (1993), Environmental Policy when Market Structure and Plant ocations are Endogenous, Journal of Environmental Economics and Management, 24, 169-86.

[14] Markusen, J.R., E.R. Morey and N. Olewiler (1995), Competition in Regional Environmental Policies when Plant ocations are Endogenous, Journal of Public Economics, 56, 55-77.

[15] Motta, M. and J-F Thisse (1994), Does Environmental Dumping ead to Delocation?, European Economic Review, 38, 563-576.

[16] Rauscher, M. (1995), Environmental Regulation and the ocation of Polluting Industries, International Tax and Public Finance, 2, 229-244.

[17] Xepapadeas, A. (1992), Environmental Policy, Adjustment Costs, and Behavior of the Firm, Journal of Environmental Economics and Management, 23 (3), 258-75.

[18] Xepapadeas, A. (1997), Advanced Principles in Environmental Policy, Aldershot: Edward Elgar.

[19] Xepapadeas, A. (1997), Economic Development and Environmental Pollution: Traps and Growth, Structural Change and Economic Dynamics, $8,327-350$

[20] Xepapadeas, A. (1998), Optimal Resource Development and Irreversibilities: Cooperative and Noncooperative Solutions, Natural Resource Modelling, 11(4), 357-377. 
</ref_section> 


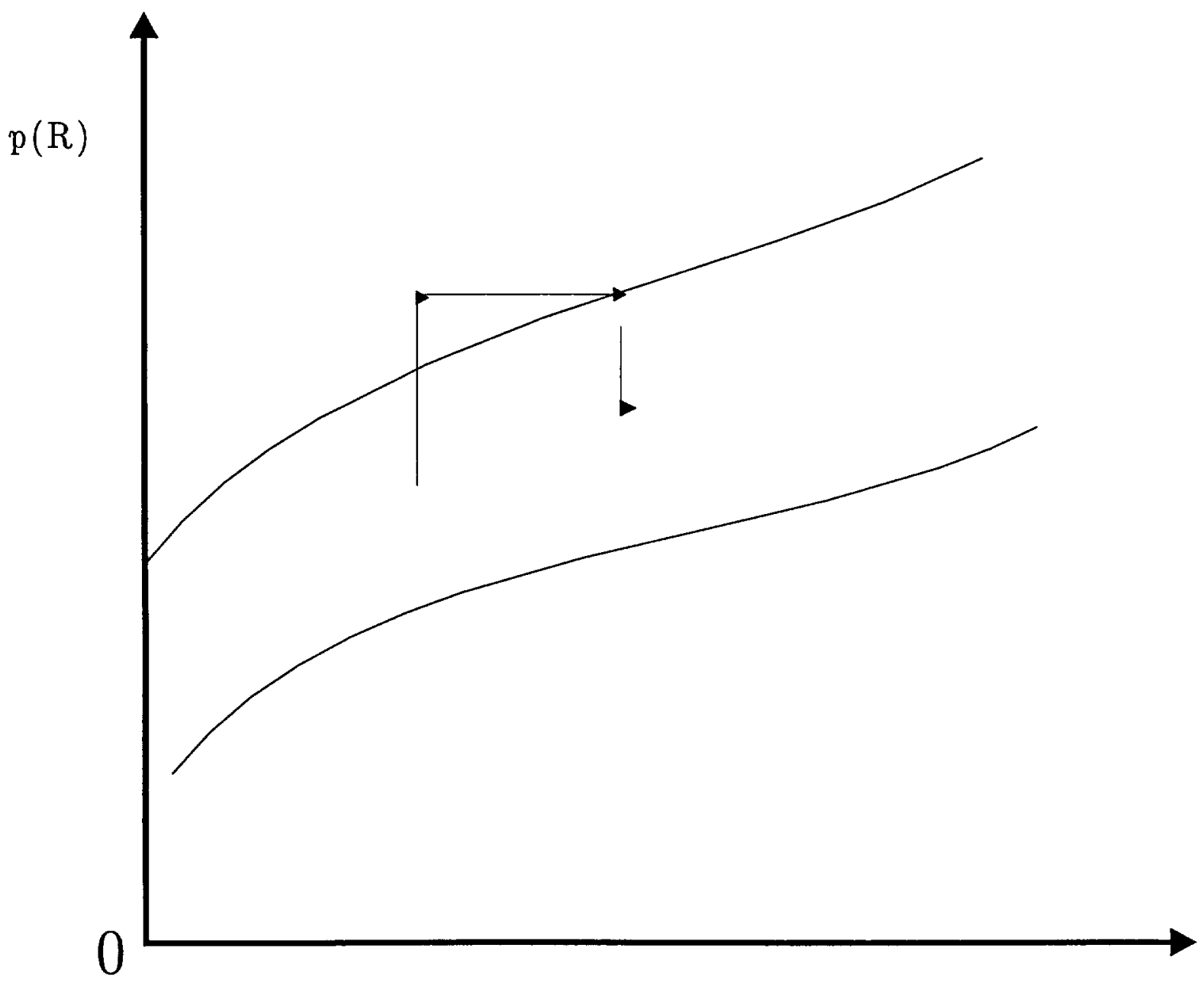

R

Figure 1: Free boundary under price uncertainty 


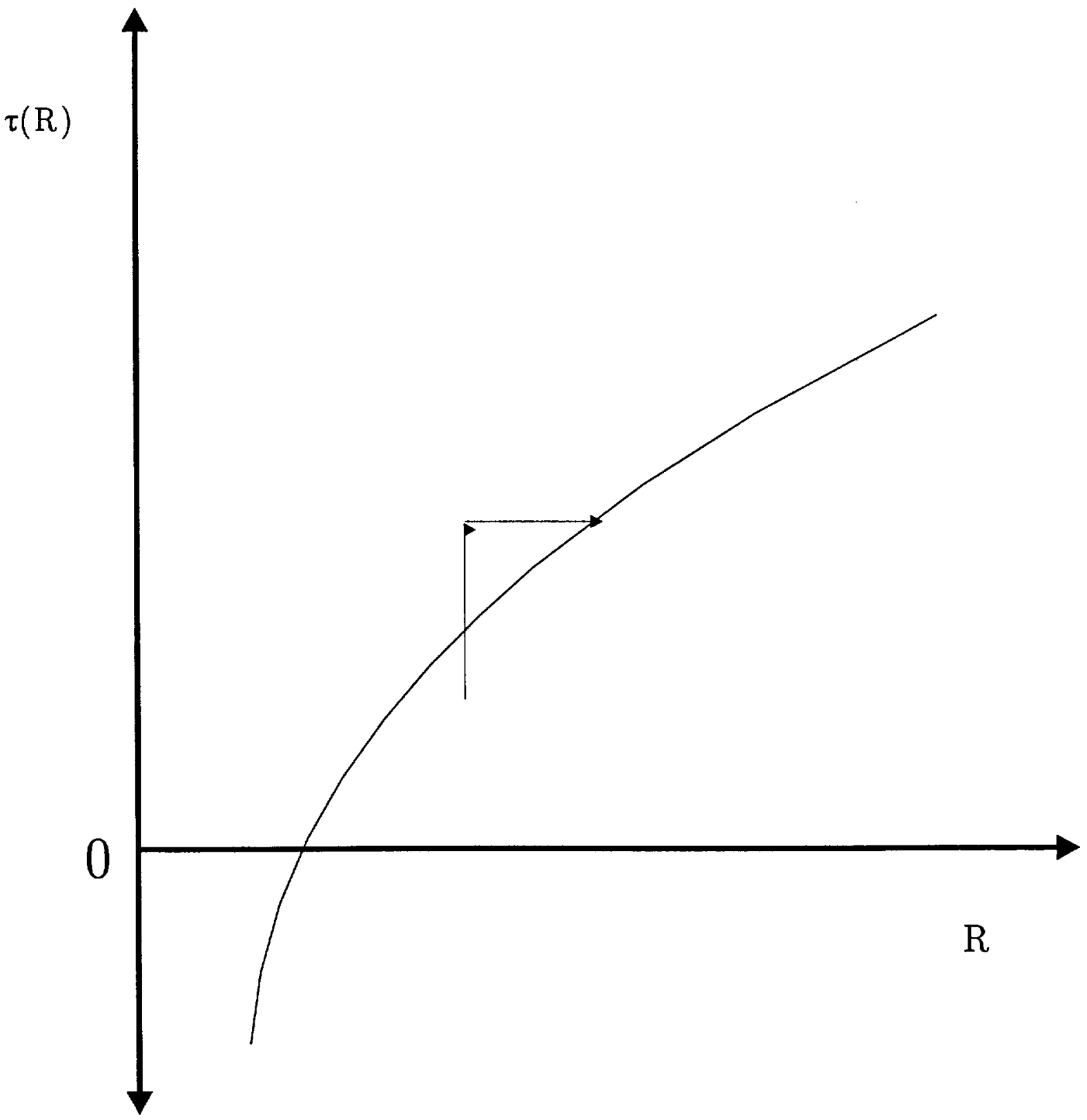

Figure 2: Free boundary under policy uncertainty 


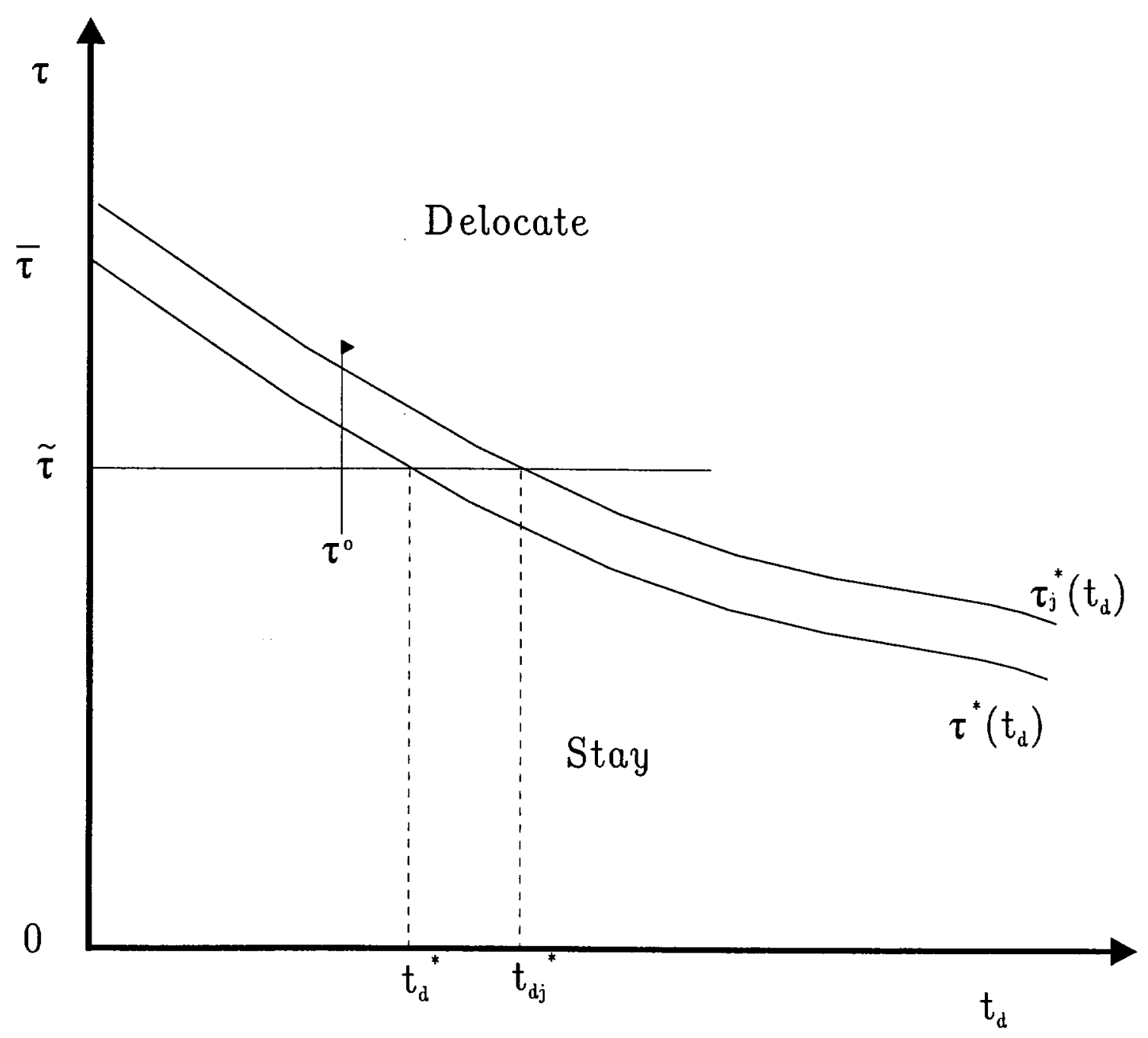

Figure 3: Threshold policy parameter 


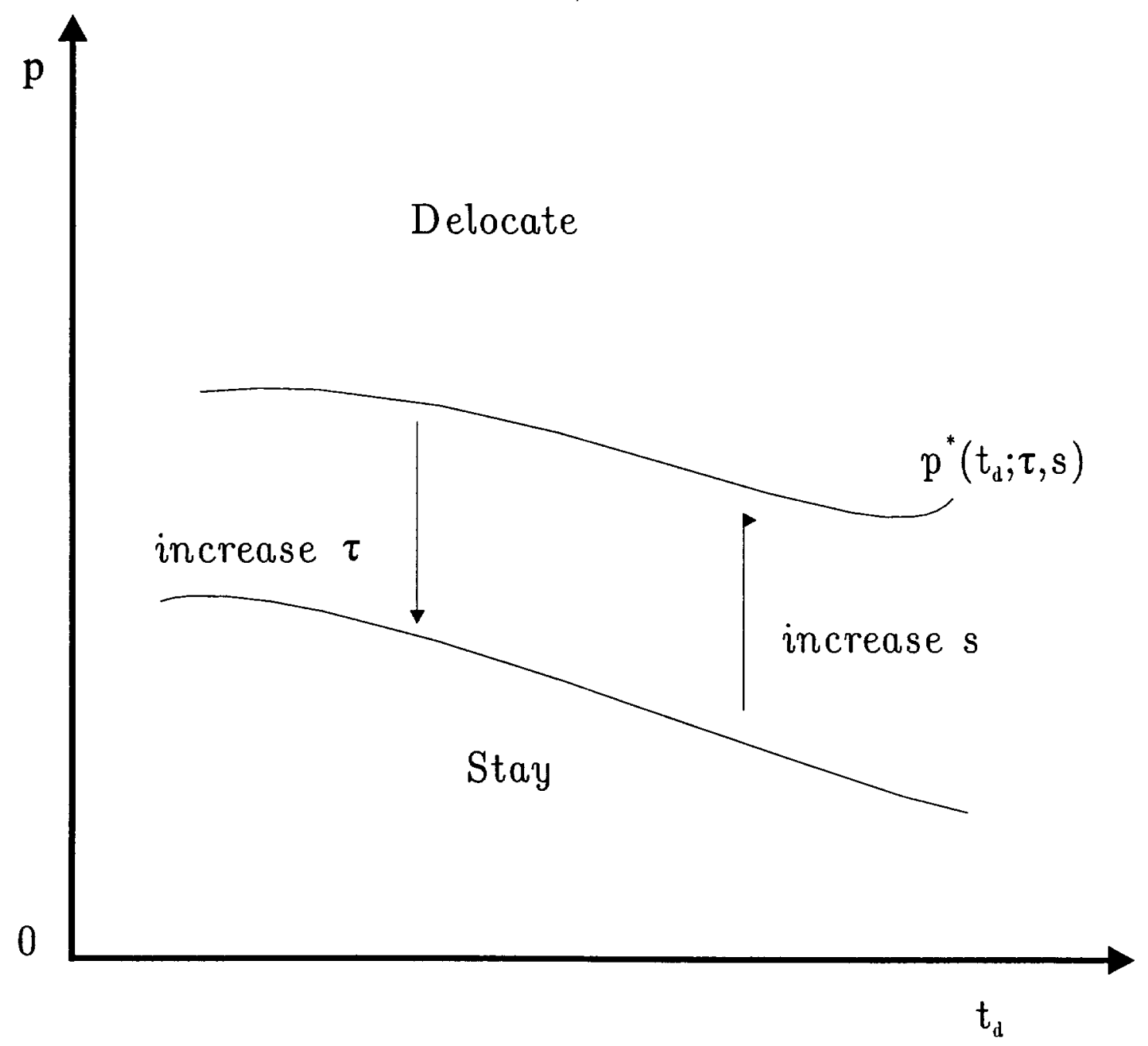

Figure 4: Relocation time policy 